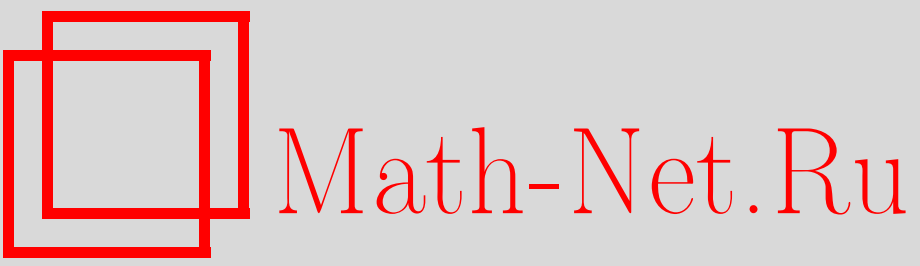

А. А. Сабыканов, Й. Микеш, П. Пешка, Симметрические, полусимметрические и рекуррентные проективно евклидовы пространства, Итоги науки и техн. Сер. Соврем. мат. и ее прил. Темат. обз., 2020, том 179, 60-66

DOI: https://doi.org/10.36535/0233-6723-2020-179-60-66

Использование Общероссийского математического портала Math-Net.Ru подразумевает, что вы прочитали и согласны с пользовательским соглашением http://www.mathnet.ru/rus/agreement

Параметры загрузки:

IP: 44.207 .124 .84

26 апреля 2023 г., $16: 36: 17$ 


\title{
СИММЕТРИЧЕСКИЕ, ПОЛУСИММЕТРИЧЕСКИЕ И РЕКУРРЕНТНЫЕ ПРОЕКТИВНО ЕВКЛИДОВЫ ПРОСТРАНСТВА
}

\author{
(c) 2020 г. А. С. САБЫКАНОВ, Й. МИКЕШ, П. ПЕШКА \\ Посвящается профессору В. Т. Базылеву
}

\begin{abstract}
АннотАция. В настоящей статье изложены некоторые результаты, полученные для симметрических, полусимметрических и полусимметрических рекуррентных проективно-евклидовых пространств. Найдены компоненты объектов аффинной связности изучаемых пространств.
\end{abstract}

Ключевые слова: симметрическое пространство, полусимметрическое пространство, рекуррентное пространство, эквиаффинное пространство, проективно-евклидово пространство.

\section{SYMMETRIC, SEMISYMMETRIC, AND RECURRENT PROJECTIVELY EUCLIDEAN SPACES}

\author{
(c) 2020 A. S. SABYKANOV, J. MIKEŠ, P. PEŠKA
}

\begin{abstract}
In this paper, we present some results obtained for symmetric, semisymmetric, and semisymmetric recurrent projectively-Euclidean spaces. Components of objects of affine connections of these spaces are found.
\end{abstract}

Keywords and phrases: symmetric space, semisymmetric space, recurrent space, equiaffine space, projective-Euclidean space.

AMS Subject Classification: 53B05, 53B20, 53B22, $11 \mathrm{Y} 11$

1. Введение. Как известно (см. [9]), пространство аффинной связности $A_{n}$ называется проективно-евклидовым, если любая геодезическая линия этого пространства отображается на прямую линию евклидова пространства $E_{n}$. В работе изложены результаты исследований симметрических, рекуррентных и полусимметрических пространств, которые являются одновременно проективно-евклидовыми.

Симметрические пространства впервые ввел в рассмотрение в 1925 г. П. А. Широков (см. [15, 16]), затем они исследовались многими авторами, например, Э. Картаном (см. [19]), А. Лихнеровичем (см. [28]). Симметрические проективно-евклидовы пространства изучал П. А. Широков (см. $[15,16])$. Геодезические отображения симметрических, полусимметрических и полусимметрических рекуррентных пространств изучали Н. С. Синюков (см. [12]), Й. Микеш (см. [6, 7]) И. Гинтерлейтнер и Й. Микеш (см. [21], а также [13, 14,29, 32, 33]).

Специальные отображения симметрических пространств и им близкие проблемы изучались, например, в $[1,2,17,29,30,34,39,40]$.

В работах И. Гинтерлейтнер и Й. Микеша [22-26] исследуются общие закономерности геодезических отображений, в частности, доказано, что геодезические отображения сохраняют класс

\footnotetext{
Работа выполнена при поддержке гранта Университета им. Ф. Палацкого (Оломоуц, Чехия) «Алгебраические и геометрические структуры», IGA PrF 2019015.
} 
гладкости метрик (псевдо-) римановых многообразий. Это свойство естественным образом обобщается для более общего случая (см. [27]).

2. Проективно-евклидовы пространства. Приведем основные понятия теории геодезических отображений пространств аффинной связности (см. например, $[10,13,20,33])$.

В пространстве аффинной связности без кручения $A_{n}$, отнесенном к локальной системе координат $x \equiv\left(x^{1}, x^{2}, \ldots, x^{n}\right)$, при помощи объекта аффинной связности $\Gamma_{i j}^{h}(x), i, j, k, \ldots=1,2, \ldots, n$, определяют тензоры Римана, Риччи и проективной кривизны Вейля:

$$
\begin{gathered}
R_{i j k}^{h}=\partial_{i} \Gamma_{k i}^{h}+\Gamma_{k i}^{\alpha} \Gamma_{j \alpha}^{h}-\partial_{k} \Gamma_{j i}^{h}-\Gamma_{j i}^{\alpha} \Gamma_{k \alpha}^{h}, \quad R_{i j}=R_{i j \alpha}^{\alpha}, \\
W_{i j k}^{h}=R_{i j k}^{h}-\frac{1}{n-1}\left(\delta_{k}^{h} R_{i j}-\delta_{j}^{h} R_{i k}\right)+\frac{1}{n+1}\left(\delta_{i}^{h} R_{[j k]}+\frac{1}{n+1}\left(\delta_{k}^{h} R_{[i j]}-\delta_{j}^{h} R_{[i k]}\right)\right),
\end{gathered}
$$

где $\partial_{i}=\partial / \partial x_{i}, \delta_{i}^{h}$ - символы Кронекера, $[i j]$ - альтернирование без деления.

Пространство $A_{n}$ принадлежит классу гладкости $C^{r}\left(A_{n} \in C^{r}\right)$, если $\Gamma_{i j}^{h}(x) \in C^{r}$. Эквиаффинным пространством называется пространство $A_{n}$, в котором тензор Риччи симметричен $\left(R_{i j}=R_{j i}\right)$. В эквиаффинном пространстве тензор проективной кривизны упрощается следующим образом:

$$
W_{i j k}^{h}=R_{i j k}^{h}-\frac{1}{n-1}\left(\delta_{k}^{h} R_{i j}-\delta_{j}^{h} R_{i k}\right) .
$$

Пространство $A_{n}$ называется плоским (или аффинным), если в нем существует система координат $x$, в которой $\Gamma_{i j}^{h}(x)=0$. Тензорным признаком плоских пространств является равенство нулю тензора Римана. В плоском пространстве можно естественным образом ввести евклидову метрику, поэтому их называют евклидовыми и обозначают $E_{n}$.

Диффеоморфизм $f: A_{n} \rightarrow \overline{A_{n}}$ называют геодезическим отображсением, если в результате $f$ все геодезические линии $A_{n}$ переходят в геодезические линии $\bar{A}_{n}$. Необходимым и достаточным условием того, чтобы отображение $f: A_{n} \rightarrow \overline{A_{n}}$ было геодезическим, является выполнение в общей по отображению $f$ системе координат $x$ следующих условий:

$$
\bar{\Gamma}_{i j}^{h}(x)=\Gamma_{i j}^{h}(x)+\delta_{i}^{h} \psi_{j}+\delta_{j}^{h} \psi_{i},
$$

где $\Gamma_{i j}^{h}$ и $\bar{\Gamma}_{i j}^{h}$ - объекты аффинной связности пространств $A_{n}$ и $\overline{A_{n}}, \psi(x)$ - некоторый ковектор.

Тензоры Римана, Риччи и проективной связности Вейля пространств $A_{n}$ и $\overline{A_{n}}$ при геодезическом соответствии связаны следующими условиями:

$$
\begin{gathered}
\bar{R}_{i j k}^{h}=R_{i j k}^{h}+\delta_{i}^{h} \psi_{[k j]}+\delta_{k}^{h} \psi_{i j}-\delta_{j}^{h} \psi_{i k}, \\
\bar{R}_{i j}=R_{i j}+(n-1) \psi_{i j}+\psi_{[i j]}, \\
\bar{W}_{i j k}^{h}=W_{i j k}^{h},
\end{gathered}
$$

где

$$
\psi_{i j}=\psi_{i, j}-\psi_{i} \psi_{j}
$$

Здесь и далее запятой обозначаем ковариантную производную по связности пространства $A_{n}$, объекты пространства $\overline{A_{n}}$ отмечены чертой, например, $\bar{R}_{i j k}^{h}$ - тензор Римана пространства $\overline{A_{n}}$.

Пространство аффинной связности $A_{n}$ называется проективно-евклидовым пространством, если оно допускает геодезическое отображение на евклидово пространство. Известно, что $A_{n}$ $(n>2)$ является проективно-евклидовым тогда и только тогда, когда $W_{i j k}^{h}=0$. Пространство $A_{n}$ является проективно-евклидовым тогда и только тогда, когда тензор Римана имеет следующее представление:

$$
R_{i j k}^{h}=\delta_{i}^{h} \psi_{[j k]}+\delta_{j}^{h} \psi_{i k}-\delta_{k}^{h} \psi_{i j}
$$

где $\psi_{i j}$ - некоторый тензор, необходимо удовлетворяющий условию

$$
\psi_{i j, k}=\psi_{i k, j}
$$

учитывая формулы (1), заключаем, что этот тензор необходимо имеет строение (2).

Тензор Риччи проективно-евклидова пространства имеет вид

$$
R_{i j}=(n-1) \psi_{i j}-\psi_{[i j]} .
$$


Проективно-евклидово пространство является эквиаффинным тогда и только тогда, когда

$$
\psi_{i j}=\psi_{j i}
$$

3. Аффинные отображения. С точки зрения пространств аффинной связности имеют большое значение аффинные отображения (см. [13,20,33]).

Отображение $f: A_{n} \rightarrow \overline{A_{n}}$ называется аффинным, если оно является геодезическим, и при этом канонический параметр геодезической линии прообраза является каноническим параметром образа (см. [33]).

Отображение $f: A_{n} \rightarrow \overline{A_{n}}$ является аффинным тогда и только тогда, когда в общей по отображению системе координат $x$ выполняются условия $\bar{\Gamma}_{i j}^{h}(x)=\Gamma_{i j}^{h}(x)$, т.е. аффинные связности тождественны и пространства $A_{n}$ и $\overline{A_{n}}$ совпадают.

Пусть пространства $A_{n}$ и $\overline{A_{n}}$ отнесены к системам координат $x$ и $\bar{x}$. Отображение

$$
f: \bar{x}^{h}=\bar{x}^{h}\left(x^{1}, x^{2}, \ldots, x^{h}\right)
$$

является аффинным тогда и только тогда, когда выполняются уравнения

$$
\begin{aligned}
\partial_{i} \bar{x}^{h} & =\bar{x}_{i}^{h} \\
\partial_{j} \bar{x}_{i}^{h} & =\Gamma_{i j}^{\alpha}(x) \bar{x}_{\alpha}^{h}-\bar{\Gamma}_{\alpha \beta}^{h}(\bar{x}(x)) \bar{x}_{i}^{\alpha} \bar{x}_{j}^{\beta}
\end{aligned}
$$

при условии $\left|\bar{x}_{i}^{h}\right| \neq 0$.

С другой стороны, если для заданных пространств $A_{n}$ и $\overline{A_{n}}$ найдено решение системы уравнений (5) относительно неизвестных функций $\bar{x}^{h}(x)$ и $\bar{x}_{i}^{h}(x)$, то тем самым построено аффинное соответствие (4) между $A_{n}$ и $\overline{A_{n}}$. В этом случае уравнения (5) представляют собой систему дифференциальных уравнений в частных производных типа Коши относительно неизвестных функций $\bar{x}^{h}(x)$ и $\bar{x}_{i}^{h}(x)$. Условие интегрируемости уравнений (4) имеет вид

$$
\bar{x}_{\alpha}^{h} R_{i j k}^{h}=\bar{R}_{\alpha \beta \gamma}^{h} \bar{x}_{i}^{\alpha} \bar{x}_{j}^{\beta} \bar{x}_{k}^{\gamma} .
$$

4. Симметрические проективно-евклидовы пространсва. П. А. Широков изучал (см. $[15,16])$ симметрические проективно-евклидовы пространства и нашел в некоторой системе координат все связности таких пространств. Однако, как покажем ниже, дополнительным исследованием устанавливаем, что каноническую форму таких пространств, полученную П. А. Широковым, можно значительно упростить.

Симметрические пространства $A_{n}$ характеризуются условием

$$
R_{i j k, l}^{h}=0
$$

на тензор Римана. П. А. Широков установил, что в любом не плоском симметрическом пространстве $A_{n}$ существует проективная система координат $x$, в которой объект связности имеет представление

$$
\Gamma_{i j}^{h}(x)=\delta_{i}^{h} \partial_{j} \Psi+\delta_{j}^{h} \partial_{i} \Psi
$$

где

$$
\Psi \equiv-\ln \sqrt{\varphi}, \quad \varphi \equiv a_{\alpha \beta} x^{\alpha} x^{\beta}+b_{\alpha} x^{\alpha}+c,
$$

$a_{i j}, b_{i}, c$ - некоторые постоянные, $a_{i j}=a_{j i} \neq 0$.

Учитывая строение функции $\varphi$, кажется, что семейство всех симметрических проективно-евклидовых пространств зависит от $(n+1)(n+2) / 2$ параметров. Однако все эти параметры фактически не существенны. Имеет место следующая теорема.

Теорема 1 (см. [11]). В любом не плоском симметрическом проективно-евклидовом пространстве $A_{n}$ существует проективная система координат $x$, в которой объект аффинной связности имеет представление

$$
\Gamma_{i j}^{h}(x)=\delta_{i}^{h} \partial_{j} \Psi+\delta_{j}^{h} \partial_{i} \Psi
$$

где

$$
\Psi=-\ln \sqrt{\varphi}, \quad \varphi=e_{1} x^{1^{2}}+e_{2} x^{2^{2}}+\ldots+e_{k} x^{k^{2}}+1, \quad e_{\tau}= \pm 1, \tau=1,2, \ldots, k .
$$


Доказательство. Доказательство вытекает из того, что легко построить линейное преобразование координат $\bar{x}^{h}=A_{\alpha}^{h} x^{\alpha}+B^{h}$, где $A_{i}^{h}, B^{h}$ - постоянные, и $\operatorname{det} A_{i}^{h} \neq 0$, при котором аффинная связность (7) примет вид (8). Это преобразование координат является аффинным.

Оказывается, что в указанном пространстве тензор $\psi_{i j}$ ковариантно постоянный и, как показал А. П. Широков, единственный, с точностью до постоянного множителя, симметричный ковариантно постоянный тензор. Этот тензор в системе координат (8) в некоторой начальной точке $x_{0}$ имеет вид

$$
\psi_{i j}\left(x_{0}\right)=\operatorname{diag}\left(e_{1}, e_{2}, \ldots, e_{k}, 0, \ldots, 0\right) .
$$

Поэтому имеет место следующее утверждение.

Теорема 2. Симметрическое проективно-евклидово пространство $A_{n}$ однозначно определяется сигнатурой симметрического ковариантно постоянного тензорного поля этого пространства.

5. Полусимметрические проективно-евклидовы пространства. Условия интегрируемости соотношений (6) имеют вид

$$
R_{i j k, l m}^{h}-R_{i j k, m l}^{h}=0 .
$$

На основании тождеств Риччи условия (9) запишутся в следующей форме:

$$
R_{\alpha j k}^{h} R_{i l m}^{\alpha}+R_{i \alpha k}^{h} R_{j l m}^{\alpha}+R_{i j \alpha}^{h} R_{k l m}^{\alpha}-R_{i j k}^{\alpha} R_{\alpha l m}^{h}=0 .
$$

Эти условия в неявном виде встречаются в работах П. А. Широкова (см. $[15,16])$ и Э. Картана (см. [19]) при изучении симметрических пространств. Затем эти условия встречаются в работах А. Г. Волкера (см. [44]) и Н. С. Синюкова (см. $[12,13])$. Пространства, в которых выполняются условия (9), Н. С. Синюков назвал полусимметрическими.

Изучению указанных пространств посвящено много исследований, например, [3-6,8]. Особый интерес к полусимметрическим пространствам вызвала гипотеза К. Номидзу (см. [35]), которая была затем отвергнута (см. [38,43]). Римановы и псевдоримановы пространства классифицировал 3. Сабо (см. $[41,42])$.

Геодезические отображения полусимметрических римановых пространств изучались в работах Н. С. Синюкова (см. [12]) и Й. Микеша (см. [6,7], а также [13,14,29, 32, 33]).

Нами доказана следующая теорема.

Теорема 3 (см. [11]). Проективно-евклидово пространство $A_{n}$ является полусимметрическим тогда и только тогда, когда оно является эквиаффинным.

Кроме того, установлено, что связность полусимметрического проективно-евклидова пространства имеет в проективной системе координат следующее представление:

$$
\Gamma_{i j}^{h}=\delta_{i}^{h} \psi_{j}+\delta_{j}^{h} \psi_{i}
$$

где $\psi=\partial_{i} \Psi, \Psi$ - некоторая функция класса $C^{2}$.

6. Полусимметрические рекуррентные проективно-евклидовы пространства. Пространства аффинной связности $A_{n}$, в которых тензор Римана абсолютно рекуррентен, Г. С. Руз назвал рекуррентными (см. [36]). Рекуррентные пространства характеризуются следующими условиями:

$$
R_{i j k, l}^{h}=\varphi_{l} R_{i j k}^{h}
$$

где $\varphi_{l}$ - некоторый ковектор. Будем считать, что этот вектор ненулевой; в противном случае бы пространство было симметрическое. Отметим, что А. Г. Волкер доказал (см. [44]), что все рекуррентные римановы пространства являются полусимметрическими.

Непосредственным образом можно показать справедливость следующей теоремы.

Теорема 4 (см. [37]). В рекуррентном проективно-евклидовом пространстве $A_{n}$ тензор $\psi_{i j}$, участвуюший в условиях (3), удовлетворяет условиям

$$
\psi_{i j, k}=\psi_{i j} \varphi_{k}, \quad \psi_{i j}=\varkappa_{i} \varphi_{j}
$$

где $\varkappa$-некоторый ненулевой вектор. 
Детальным анализом уравнений (3) была установлена справедливость следующей теоремы.

Теорема 5 (см. [37]). Пространство аффинной связности $A_{n}$ является полусимметрическим рекуррентным проективно-евклидовым пространством тогда и только тогда, когда его тензор Римана имеет вид (3), и при этом имеют место соотношения

$$
\begin{aligned}
\psi_{i j} & =\varkappa \varphi_{i} \varphi_{j}, \\
\varphi_{i, j} & =\frac{1}{2}\left(1-\frac{\varkappa^{\prime}}{\varkappa}\right) \varphi_{i} \varphi_{j},
\end{aligned}
$$

где $\varkappa$-некоторая функиия аргумента $\varphi$.

Изучая аффинные трансформации координат (4), мы установили следующий факт.

Теорема 6. В любом неплоском полусимметрическом рекуррентном пространстве аффинной связности $A_{n}$ существует проективная система координат х, в которой обгект аффинной связности имеет следующее представление:

$$
\Gamma_{i j}^{h}=s\left(x^{1}\right) \cdot\left(\delta_{i}^{h} \delta_{j}^{1}+\delta_{j}^{h} \delta_{i}^{1}\right),
$$

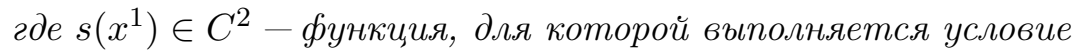

$$
\left(\ln \left(s^{\prime}-s^{2}\right)\right)^{\prime} \neq 4 s .
$$

7. Пример компактного полусимметрического рекуррентного пространства аффинной связности, которое является проективно евклидовым. Построим компактное полусимметрическое рекуррентное пространство аффинной связности $A_{n}$, которое является гомеоморфным $n$-мерному тору.

Пусть $A_{n}=S^{1} \times S^{1} \times \ldots \times S^{1}$ и $x^{1}, x^{2}, \ldots, x^{n}$ - соответствующие углы окружностей $S^{1}$. Имеем векторные поля $X_{1}=\partial_{1}, X_{2}=\partial_{2}, \ldots, X_{n}=\partial_{n}$, построенные «в целом». Построим аффинную связность «в целом», для которой

$$
\nabla_{X_{i}} X_{j}=\Gamma_{i j}^{k} \cdot X_{k}=s\left(x^{1}\right) \cdot\left(\delta_{i}^{1} X_{j}+\delta_{j}^{1} X_{i}\right)
$$

где $s\left(x^{1}\right)$ - дифференцируемая функция, определенная на $S^{1}$.

В силу строения коэффициентов аффинной связности (11) пространство является полусимметрическим рекуррентным пространством аффинной связности, которое проективно евклидово, а в силу глобального построения многообразия - компактно.

Этот пример построен подобным образом, как в [31] построены компактные пространства, содержащие конциркулярные векторные поля.

\section{СПИСОК ЛИТЕРАТУРЫ}

1. Березовский В. Е., Гусева Н. И., Микеш Й. О частном случае почти геодезических отображений первого типа пространств аффинной связности, при котором сохраняется некоторый тензор// Мат. заметки. - 2015. - 98, № 3. - С. 463-466.

2. Березовский В. Е., Ковалев Л. Е., Микеш Й. О сохранении тензора Римана относительно некоторых отображений пространств аффинной связности// Изв. вузов. Мат. - 2018. - № 9. - С. 3--10.

3. Кайгородов B. Р. Структура кривизны пространства-времени// Итоги науки и техн. Сер. Пробл. геом. - 1983. - 14. - C. 177--204.

4. Ковалев П. И. Тройные системы Ли и пространства аффинной связности// Мат. заметки. - 1973. 14, № 1. - С. 107--112.

5. Лумисте Ю. Г. Полусимметрические подмногообразия// Итоги науки и техн. Сер. Пробл. геом. 1991. - 23. - С. 3- -28 .

6. Микеш Й. Геодезические отображения полусимметрических пространств// Деп. в ВИНИТИ. - 1976. - № 3924-76Деп..

7. Микеш Й. Геодезические отображения на полусимметрические пространства// Изв. вузов. Мат. 1994. - № 2. - С. 37-43.

8. Мирзоян B. A. Классификация Ric-полупараллельных гиперповерхностей в евклидовых пространствах// Мат. сб. - 2000. - 191, № 9. - С. 65--80. 
9. Норден А. П. Пространства аффинной связности. - М.: Наука, 1976.

10. Петров А. З. Новые методы в общей теории относительности. - М.: Наука, 1966.

11. Сабыканов А. А., Микеш Й., Пешка П. О полусимметрических проективно евклидовых пространствах// Сб. тр. Междунар. молодежн. шк.-семин. и Междунар. науч. конф. «Современная геометрия и ее приложения» (Казань, 27 ноября-3 декабря 2017 г.). - Казань: КФУ, 2017. - С. $123-125$.

12. Синюков Н. С. О геодезическом отображении римановых пространств на симметрические римановы пространства// Докл. АН СССР. - 1954. - 98, № 1. - С. 21-23.

13. Синюков Н. С. Геодезические отображения римановых пространств. - М.: Наука, 1979.

14. Синюков Н. С. Почти геодезические отображения аффинно-связных и римановых пространств// Итоги науки и техн. Сер. Пробл. геом. - 1982. - 13. - С. 3--26.

15. Широков П. А. Постоянные поля векторов и тензоров в римановых пространствах// Изв. Казанск. физ.-мат. о-ва. - 1925. - № 25. - С. 86-114.

16. Широков П. А. Избранные работы по геометрии. - Казань: Изд. Казанск. ун-та, 1966.

17. Berezovskii V., Hinterleitner I., Mikeš J. Geodesic mappings of manifolds with affine connection onto the Ricci symmetric manifolds// Filomat. — 2018. — 32, № 2. — P. 379-385.

18. Cartan É. Sur les variété à connexion projective// Bull. Soc. Math. Fr. — 1924. — 52. — P. $205-241$.

19. Cartan É. Sur une classe remarquable d'espaces de Riemann, II// Bull. Soc. Math. Fr. — 1927. — 55. — P. 114-134.

20. Eisenhart L. P. Non-Riemannian geometry. - Princeton Univ. Press, 1926.

21. Hinterleitner I., Mikeš J. Geodesic mappings onto Weyl manifolds// J. Appl. Math. — 2009. — 2, № 1. — P. $125-133$.

22. Hinterleiner I., Mikěs J. Fundamental equations of geodesic mappings and their generalizations// J. Math. Sci. - 2011. - 174. - P. 537-554.

23. Hinterleitner I., Mikeš J. Projective equivalence and spaces with equi-affine connection// J. Math. Sci. 2011. - 177. - P. 546-550.

24. Hinterleitner I., Mikěs J. Geodesic mappings and Einstein spaces// in: Geometric Methods in Physics. Basel: Birkhäuser, 2013. - P. 331-335.

25. Hinterleiner I., Mikěs J. Geodesic Mappings of (pseudo-) Riemannian manifolds preserve class of differentiability// Miskolc. Math. Notes. - 2013. - 14. - P. 89-96.

26. Hinterleitner I., Mikěs J. Geodesic mappings and differentiability of metrics, affine and projective connections// Filomat. - 2015. - 29. - P. 1245-1249.

27. Hinterleiner I., Mikěs J. Geodesic mappings onto Riemannian manifolds and differentiability// in: Geometry, Integrability and Quantization. — Sofia: Bulgar. Acad. Sci., 2017. — Vol. 18. — P. 183-190.

28. Lichnerowicz A. Courbure, nombres de Betti, et espaces symétriques// Proc. Int. Congr. Math. (Cambridge, Massachusetts, August 30-September 6, 1950). - Am. Math. Soc., 1952. — P. 216-223.

29. Mikěs J. Geodesic mappings of affine-connected and Riemannian spaces// J. Math. Sci. — 1996. — 78. — P. 311-333.

30. Mikeš J., Berezovski V. E., Stepanova E., Chudá H. Geodesic mappings and their generalizations// J. Math. Sci. - 2016. - 217. - P. 607-623.

31. Mikeš J., Chodorová M. On concircular and torse-forming vector fields on compact manifolds// Acta Math. Acad. Paedagog. Nyházi. — 2010. — 26. — P. 329-335.

32. Mikeš J., Vanžurová A., Hinterleitner I. Geodesic mappings and some generalizations. — Olomouc: Palacky Univ. Press, 2009.

33. Mikeš J. et al. Differential geometry of special mappings. - Olomouc: Palacky Univ. Press, 2015.

34. Najdanović M., Zlatanović M., Hinterleitner I. Conformal and geodesic mappings of generalized equidistant spaces // Publ. Inst. Math. Beograd. - 2015. — 98 (112). — P. 71-84.

35. Nomizu K. On hypersurfaces satisfying a certain condition on the curvature tensor// Tôhoku Math. J. 1968. - 20. - P. 46-59.

36. Ruse H. S. On simply harmonic spaces// J. London Math. Soc. - 1947. — 21. — P. $243-247$.

37. Sabykanov A., Mikeš J., Peška P. Recurrent equiaffine projective-Euclidean spaces// Filomat. — 2019. — 33, № 4.

38. Sekigawa $K$. On some hypersurfaces satisfying $R(X, Y) \cdot R=0 / /$ Tensor. -1972 . $-25 .-$ P. $133-136$.

39. Stanković M., Mincić S., Velimirović Lj., Zlatanović M. On equitorsion geodesic mappings of general affine connection spaces// Rend. Semin. Mat. Univ. Padova. — 2010. — 124. — P. 77-90. 
40. Stepanov S., Shandra I., Mikěs J. Harmonic and projective diffeomorphisms// J. Math. Sci. — 2015. — 207. - P. 658-668.

41. Szabó Z. I. Structure theorems on Riemannian spaces satisfying $R(X, Y) \cdot R=0$. I. The local version// J. Differ. Geom. - 1983. - 17. - P. 531-582.

42. Szabó Z. I. Structure theorems on Riemannian spaces satisfying $R(X, Y) \cdot R=0$. II. Global versions// Geom. Dedic. - 1985. - 19. - P. 65-108.

43. Takagi $H$. An example of Riemannian manifolds satisfying $R(X, Y) \cdot R=0$ but not $\nabla R=0 / /$ Tôhoku Math. J. - 1972. - 24. - P. 105-108.

44. Walker A. G. On Ruse's spaces of recurrent curvature// Proc. London Math. Soc. — 1950. — 52. P. 36-64.

Сабыканов Алмаз Асанович

Киргизский национальный университет, Бишкек, Киргизская Республика

E-mail: almazbek.asanovich@mail.ru

Микеш Йозеф

Университет им. Ф. Палацкого, Оломоуц, Чехия

E-mail: josef .mikes@upol.cz

Пешка Патрик

Университет им. Ф. Палацкого, Оломоуц, Чехия

E-mail: patrik.peska@upol.cz 